group. Two ICDs have been removed/deactivated after exclusion of a known familial mutation.

\section{THE UNITED KINGDOM TRANSCATHETER AORTIC VALVE REGISTRY - OUTCOMES TO DECEMBER 2009 AND UPDATE}

doi:10.1136/heartjnl-2011-300198.163

P F Ludman. On Behalf of the UK TAVI Steering Group, Birmingham, UK

Introduction The United Kingdom Transcatheter Aortic Valve (TAVI) Registry was established to define the clinical and procedural details of all patients being treated by TAVI, regardless of access route or technology used, and to assess their outcomes. The registry has captured all cases in England and Wales.

Methods For every TAVI performed, all centres complete an agreed dataset. The data are encrypted and sent electronically to servers at the Central Cardiac Audit Database (CCAD) for analysis. A unique patient identifier (the NHS number) is used to link with the NHS Central Register to track mortality. This analysis is based on all procedures performed up to 31st December 2009

Results 25 centres in England and Wales performed a total of 877 procedures in 870 patients; 66 in 2007, 273 in 2008 and 538 in 2009. Median number of cases per centre was 24. Median (IOR) age was 82 (78-87) yrs. 52\% were male, and mean logistic Euroscore (LES) was $22.2 \%$. $68 \%$ were transfemoral, the remainder being mainly transapical. $90 \%$ of CoreValve implants and $46 \%$ of Edwards used a transfemoral approach. Patients needing a transapical route had more comorbid conditions than those treated by a transfemoral route (LES $25.2 \%$ vs $20.9 \%$ ). Mortality tracking was successful in $100 \%$ of patients. Survival at 30 days was $93.1 \%, 78.9 \%$ at 1 year (443 at risk) and $72.3 \%$ at 2 years (114 at risk). Survival was significantly poorer in those needing non-transfemoral approaches (1 year survival $73.5 \%$ vs $81.4 \%$ ). Survival was also poorer in those with poorer LV ejection fraction, with moderate or severe post procedural aortic regurgitation and with a LES $>40$. Survival was not associated with age, NYHA class, or the presence of concomitant coronary artery disease, and not different between those with a LES $<21$ compared with LES 21-40. There was also no difference in survival between patients treated with CoreValve or Edwards technologies, or between proctored and non proctored cases. There was a significant improvement in survival over the 3 years of the registry, and a change in demographics with the proportion of patients with prior CABG rising from $16.4 \%$ in 2007 to $29.9 \%$ in 2009. The total number of cases in the UK TAVI registry has risen to 1490 as of 29 Nov 2010. More details of the 2010 cohort will be available at the time of presentation (Abstract 163 figure 1).

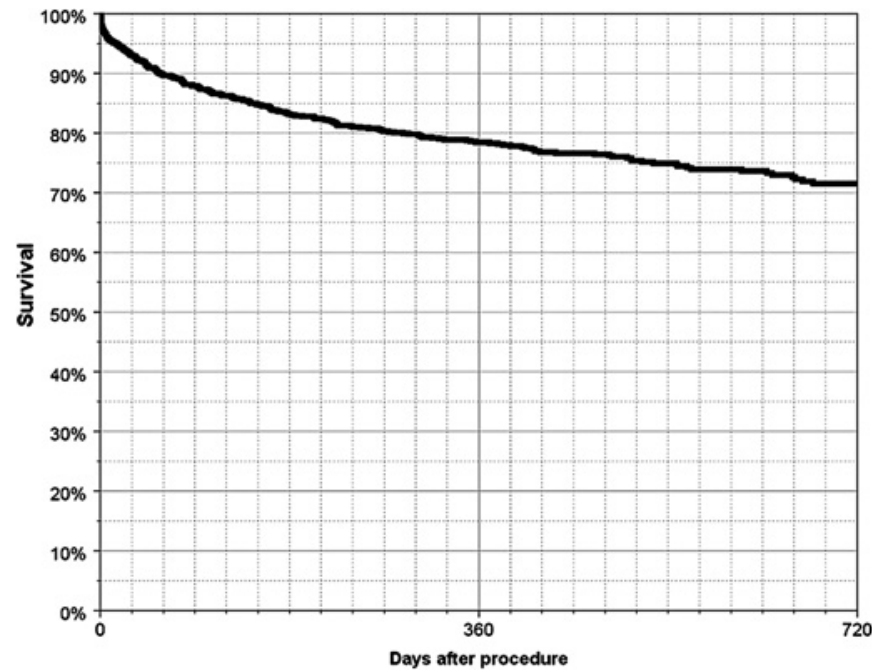

Abstract 163 Figure 1
Conclusions The UK TAVI Registry has successfully captured the entire TAVI activity of England and Wales incorporating the learning curves of every centre. Outcomes following TAVI are excellent in a high risk patient population. Outcomes are better in the population who are suitable for a transfemoral approach (with less comorbidity) than those treated with the transapical approach. These data suggest that careful proctoring allows the introduction of a new treatment method without an adverse effect on patient outcome, and we have demonstrated no systematic difference in outcome between the two commercially available technologies.

\section{EARLY HAEMODYNAMIC CHANGES AND MYOCARDIAL INJURY AFTER TRANSFEMORAL TRANSCATHETER AORTIC VALVE IMPLANTATION (TAVI)}

doi:10.1136/heartjnl-2011-300198.164

R Dworakowski, A Bhan, B Brickham, O Wendler, M Monaghan, A M Shah, P MacCarthy. Kings College Hospital, Kings Health Partners, London, UK

Purpose Transfemoral (TF) TAVI is a novel procedure for the treatment of severe aortic stenosis, without the need for thoracotomy or cardiopulmonary bypass. The procedure results in almost instantaneous normalisation of transvalvular gradients, but little is yet known about the periprocedural haemodynamic effects. We aimed to describe these effects using 3D and tissue Doppler (tD) transthoracic echocardiography (TTE) and Cardiac Output monitoring. Methods In 16 patients undergoing TF TAVI haemodynamics were characterised with a number of $\mathrm{tD}$ and $3 \mathrm{D}$ TTE measurements.

A

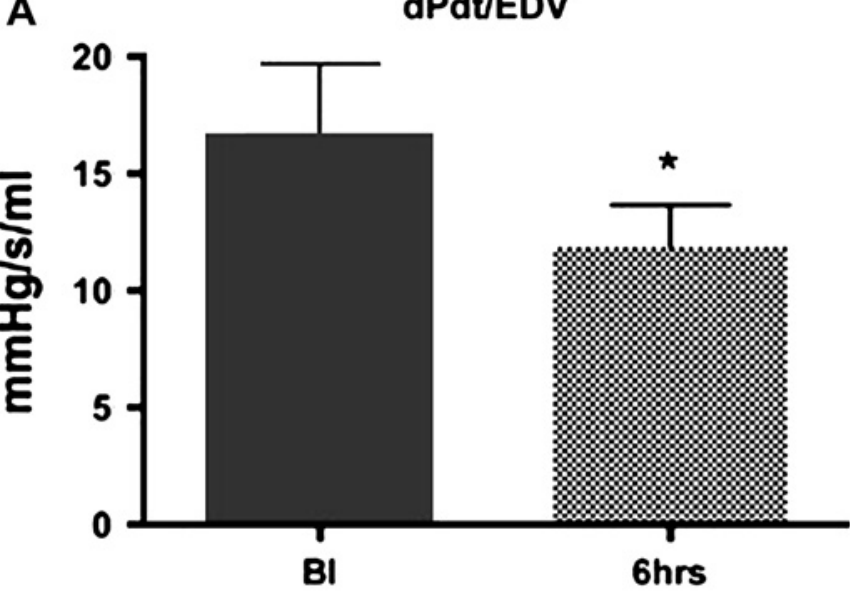

B E/E' medial

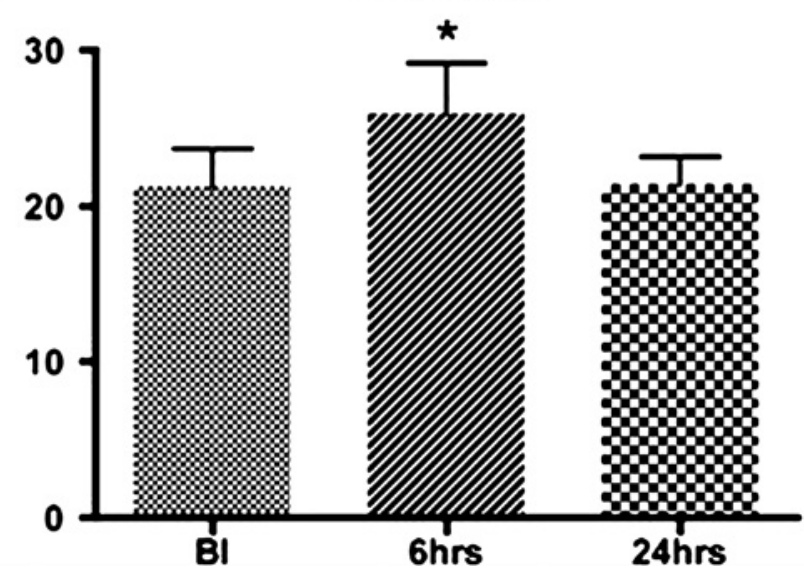

Abstract 164 Figure 1 
These were taken at multiple time points (baseline, 6 and 24 hours post procedure). Calculated volumetric parameters included $3 \mathrm{D}$ enddiastolic volume (EDV) and end-systolic volume (ESV), stroke volume (SV) and 3D LA volume (LAV). Diastolic function was monitored using the indices mean E:E' and systolic function/ contractility was measured with $\mathrm{dP} / \mathrm{dt}$ max and early peak systolic velocity $\left(\mathrm{S}^{\prime}\right)$. The FloTrac system (consisting of the Vigileo monitor and sensor), uses a clinically validated algorithm to provide continuous cardiac output (CO), stroke volume (SV) and systemic vascular resistance in real-time.

Results TAVI resulted in an immediate increase in cardiac output (3.7 (baseline), 4.6 (6 h) 4.5 l/min ( $24 \mathrm{~h}), \mathrm{p}<0.5$ baseline vs $6 \mathrm{~h}$ and $24 \mathrm{~h})$ with no significant change in systemic vascular resistance (1162, 1292 and 1367 dyn*s/cm ${ }^{5}$ ). However, $6 \mathrm{~h}$ post-TAVI there was a significant decrease in systolic function as measured by $\mathrm{dP} / \mathrm{dt}$ max/EDV (see Abstract 164 figure 1A) and co-existent impairment of diastolic function as indicated by medial E: E' values (see Abstract 164 figure $1 \mathrm{~B}$ ), which was associated with an appropriate increase in LA volume (70.3, 82.6 and $72.8 \mathrm{ml}, \mathrm{p}<0.05$ baseline vs $6 \mathrm{~h}$ ). Following this, there was a recovery of both systolic and diastolic indices. In addition, another marker of systolic function, $\mathrm{S}^{\prime}$ increased after $24 \mathrm{~h}(6.4,6.6,8.2 \mathrm{~cm} / \mathrm{s}, \mathrm{p}<0.05$ baseline vs $24 \mathrm{~h}$ and $6 \mathrm{~h}$ vs $24 \mathrm{~h})$. Concurrent with this recovery, we observed a significant decrease in EDV and ESV at $24 \mathrm{~h}$ post-TAVI (EDV: 94.9 to $83.4 \mathrm{ml}$ $(p<0.05)$; ESV 41.9 to $33.5 \mathrm{ml}(p<0.05))$. These changes in haemodynamics were associated with significant increase of troponin I levels at $24 \mathrm{~h}$ and increase in CK-MB at $6 \mathrm{~h}$ after the procedure (troponin: 0.06 vs $1.19 \mu \mathrm{g} / \mathrm{l}, \mathrm{p}<0.05$; CK-MB 1.6 vs $6.6 \mu \mathrm{g} / \mathrm{l}, \mathrm{p}<0.05$ ). Conclusion Successful TF TAVI results in an immediate improvement in cardiac output. However, overlying this, within the first $24 \mathrm{~h}$ both systolic and diastolic dysfunction occurs. The rise in the markers of myocardial injury suggest this may be due to myocardial stunning and/or some periprocedural myocardial damage. Recovery of contractility is observed after 24 hours.

\section{THE OXVALVE STUDY: ECHOCARDIOGRAPHIC SCREENING FOR VALVULAR HEART DISEASE IN THE COMIMUNITY SETTING: METHODOLOGY, FEASIBILITY AND PRELIMINARY RESULTS}

doi:10.1136/heartjnl-2011-300198.165

${ }^{1} \mathrm{~J} \mathrm{~L} \mathrm{~d}$ Arcy, ${ }^{2} \mathrm{D}$ Ebbs, ${ }^{3} \mathrm{P}$ Grimwade, ${ }^{4} \mathrm{~A} \mathrm{~J}$ Farmer, ${ }^{4} \mathrm{D}$ Mant, ${ }^{1} \mathrm{~B}$ D Prendergast. ${ }^{1} \mathrm{~J}$ ohn Radcliffe Hospital, Oxford, UK; ${ }^{2}$ Didcot Health Centre, Oxford, UK; ${ }^{3}$ Bampton Medical Practice, Oxford, UK; ${ }^{4}$ Department of Public Health and Primary Care, University of Oxford, Oxford, UK

Introduction Valvular heart disease (VHD) is poorly researched in comparison with other areas of cardiovascular disease. Principle limitations are the diverse nature of patients with VHD, inability to identify individuals at the earliest stages of disease and lack of an appropriate investigational infrastructure. Studies addressing the contemporary epidemiology and natural history of VHD are scarce but demonstrate an increasing prevalence in the elderly, associated with significant morbidity and mortality. Cohort studies in the USA are ongoing but there are no European or UK studies to date. We have developed a large scale, prospective community echocardiographic screening study within the adult Oxfordshire population, to determine the epidemiological characteristics of VHD in the UK for the first time, to assess the acceptability of echocardiographic screening for VHD, and establish cohorts with well-characterised genetic and echocardiographic phenotypes for future study. Herein, we present preliminary data for the first 1050 patients, with enrolment ongoing. Methods Patients $>65$ years, registered with participating general practices (GP) and with no known VHD, were invited to attend their GP surgery where routine demographic and cardiac data were collected and a focused examination undertaken. Participants underwent a standard transthoracic echocardiogram (TTE) according to
British Society of Echocardiography guidelines. The threshold for inclusion in the screen positive group was deliberately low to capture all manifestations of VHD. Participants were given preliminary results, before completing a shortened Spielberger STAI questionnaire. Results Uptake was 46\% (age range 65-96 years; male to female ratio $1: 1.1)$. VHD was detected in $33 \%$ of participants and prevalence increased with increasing age (see Abstract 165 figure 1). Mitral regurgitation and aortic regurgitation were the most common lesions detected (present in $17 \%$ and $14 \%$ respectively). The majority of VHD was graded as mild (84\%); only $1 \%$ of VHD detected was severe. The majority of participants (99\%) described themselves as calm or relaxed at the time of screening; none expressed significant levels of worry or tension. $98 \%$ would be prepared to undergo repeat echocardiography as screening for VHD.

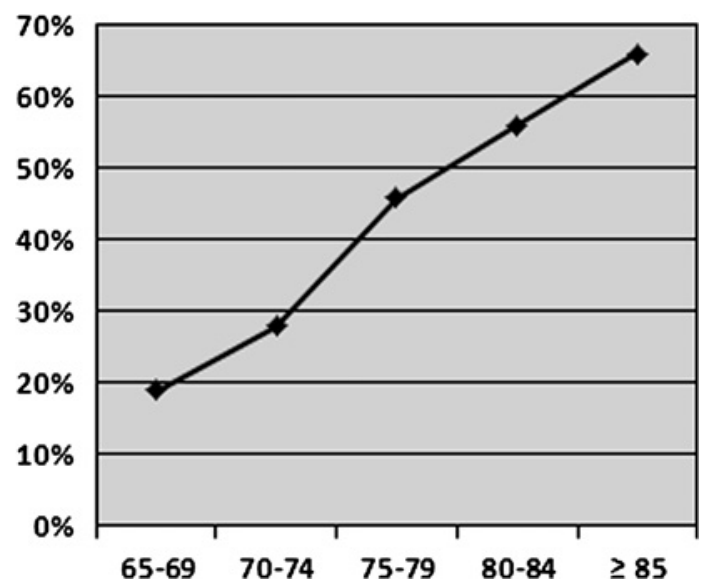

Abstract 165 Figure 1 Prevalence of VHD in $\geq 65$ s by age group.

Conclusions The prevalence of VHD in adults aged over 65 in the Oxfordshire population, using a low threshold for detection, is approximately $33 \%$ and increases with age. Mitral regurgitation is the most common lesion, and the majority of detected VHD is mild. Echocardiographic screening for VHD is feasible in the primary care setting and acceptable in this group of patients.

\section{CARDIOVASCULAR MAGNETIC RESONANCE (CMR) TAGGING IDENTIFIES DIFFERENTIAL VENTRICULAR REMODELLING IN PATIENTS WITH BICUSPID VS TRICUSPID AORTIC VALVE DISEASE}

doi:10.1136/heartjn-2011-300198.166

S Bull, J Suttie, N Blundell, J M Francis, T D Karamitsos, A Pitcher, J D'Arcy, B Prendergast, S Neubauer, S G Myerson. John Radcliffe Hospital, Oxford, UK

Background Bicuspid aortic valves (BAV) are a common inherited abnormality with a very high rate of adverse cardiac events at an earlier age than tricuspid aortic valves (TAV). Risk stratification for moderate to severe aortic stenosis, in both bicuspid and tricuspid disease, remains a significant clinical challenge. It is unknown whether pathological left ventricular (LV) remodelling, a strong predictor of adverse cardiac events, differs between patients with bicuspid and tricuspid valvular disease with comparable transvalvular gradients. Cardiovascular magnetic resonance (CMR) tagging provides detailed characterisation of global and regional contractility, and is a powerful investigative tool in the assessment of myocardial disease. We therefore assessed left ventricular strain (using CMR tagging), valve morphology and LV hypertrophy in patients with bicuspid and tricuspid aortic valve disease matched for transvalvular gradient.

Methods 42 subjects were recruited in total: 24 patients with moderate to severe BAV (age $55 \pm 15 \mathrm{yrs}$, female $21 \%$, peak trans- 